Bull. Austral. Math. Soc.

Vol. 75 (2007) [453-458]

\title{
ON THE SELF-INTERSECTIONS OF AN IMMERSED SPHERE
}

\section{ALBERT BORBÉLY}

\begin{abstract}
A closed curve $f: S^{1} \rightarrow \mathbb{R}^{2}$ in general position gives rise to a word whose letters are the self-intersection points, each of them appearing exactly twice. Such a word is called a Gauss code. The problem of determining whether a given Gauss code is realisable or not was first proposed by Gauss and it has been settled a long time ago. The analogous question for immersions $f: S^{2} \rightarrow \mathbb{R}^{3}$ in general position is settled only in a special case when the immersion has no triple points. We give a necessary condition for a system of curves to be realisable by a general immersion $f: S^{2} \rightarrow \mathbb{R}^{3}$.
\end{abstract}

\section{INTRODUCTION}

A double occurrence word over a finite alphabet is a word in which each letter appears exactly twice. A closed curve $f: S^{1} \rightarrow \mathbb{R}^{2}$ in general position (that is without self-tangencies and finitely many double crossings) gives rise to a double occurrence word whose letters are the self-intersection points, each of them appearing exactly twice, in the order as the curve is traversed once. Such a word is called a Gauss code.

A closed curve $f: S^{1} \rightarrow \mathbb{R}^{2}$ in general position is a realisation of a given Gauss code if it gives rise to the same code up to cyclic permutation. The problem whether a given word in which every letter appears exactly twice can be realised by a closed curve was first proposed by Gauss [2] and was solved by P. Rosenstiehl [5]. Since then many solutions have appeared, see for example $[1,3,6]$.

The similar problem for smooth immersions of $S^{2}$ into $\mathbb{R}^{3}$ in general position is open and it is not even well formulated. Let $f: S^{2} \rightarrow \mathbb{R}^{3}$ be a smooth immersion in general position and denote by $\Gamma_{f}$ the set of multiple points given as $\Gamma_{f}=\left\{x \in S^{2}\right.$ : $f^{-1}(f(x))$ consists of more than one point $\}$. Since the self-intersections of $f\left(S^{2}\right)$ are transversal $\Gamma_{f}$ contains only double and triple points. As a matter of fact there are finitely many smooth closed curves $\gamma_{i}: S^{1} \rightarrow S^{2}, i=1, \ldots, n$ such that $\Gamma_{f}=\bigcup_{i=1}^{n} \gamma_{i}$. All intersections of the curves $\gamma_{i}$ are transversal including self-intersections.

We call two points $p, q \in S^{2}$ conjugate if $f(p)=f(q)$ and we call two curves $\gamma_{1}:[a, b] \rightarrow S^{2}$ and $\gamma_{2}:[a, b] \rightarrow S^{2}$ conjugate if $f\left(\gamma_{1}(t)\right)=f\left(\gamma_{2}(t)\right)$ for all $t \in[a, b]$. For every $\gamma_{i}: S^{1} \rightarrow S^{2}$ there is a unique curve $\gamma_{j}: S^{1} \rightarrow S^{2}$ that is conjugate to $\gamma_{i}$.

Received 14th November, 2006

Copyright Clearance Centre, Inc. Serial-fee code: 0004-9727/07 \$A2.00+0.00. 
The Gauss code of an immersion $f: S^{2} \rightarrow \mathbb{R}^{3}$ would consist of a system of curves $\gamma_{i}: S^{1} \rightarrow S^{2}$ in general position for $i=1, \ldots, n$ together with the conjugate relations.

The analogous problem for immersed spheres would be to determine whether a given system of curves together with a conjugate relation can be realised as the inverse image of the self-intersection set of a smooth immersion. In general this problem is open and not even well formulated because it is not specified exactly what one means by the term conjugate relations. Clearly it would have to include all the obvious conditions that must be satisfied by a multiple point set of an immersion in general position.

However in a special case when the immersion has no triple points the problem can be formulated properly and in this case there is a complete characterisation [4].

The aim of this note is to look at the general case and give at least a necessary condition. It can be considered to be the generalisation of the fact - known to Gauss [2] already - that in a realisable Gauss code the length of the word between any two identical letters is even. The statement is the following.

Theorem. Let $f: S^{2} \rightarrow \mathbb{R}^{3}$ be a smooth immersion in general position and let $\gamma_{i}: S^{1} \rightarrow S^{2}$ for $i=1, \ldots, n$ be the set of self-intersection curves. Suppose that $\gamma_{1}, \gamma_{2}: S^{1} \rightarrow S^{2}$ are conjugate curves and $p_{i}=\gamma_{i}\left(t_{0}\right)$ for $i=1,2$ are conjugate points. Let $s:[0,1] \rightarrow S^{2}$ be a curve connecting $p_{1}$ and $p_{2}$ such that if $s$ intersects one of the $\gamma_{i}$ 's then the intersection is transversal. Denote by $k_{s}$ the number of intersections. If the tangent vectors $\left\{\gamma_{1}^{\prime}\left(t_{0}\right), s^{\prime}(0)\right\}$ and $\left\{\gamma_{1}^{\prime}\left(t_{0}\right), s^{\prime}(1)\right\}$ have the same orientation, then $k_{s}$ is even: otherwise it is odd.

This of course means that the parity of the number of intersections does not depend on the choice of the curve $s$.

The condition that $f: S^{2} \rightarrow \mathbb{R}^{3}$ is a smooth immersion in general position is important. Without that condition the multiple point set of the immersion can have a complicated structure. It may also have a non-empty interior. For example the multiple point set of the Boys surface (an immersion of $S^{2}$ into $\mathbb{R}^{3}$ such that the antipodal points are mapped into the same point or the immersion of the projective plain into $\mathbb{R}^{3}$ ) is the whole of $S^{2}$.

One of the possible ways to use this theorem is the following. Suppose we are given $2 n$ closed non-parameterised curves on $S^{2}$ (in general position) together with a pairing describing the conjugate pairs of curves. If this system of curves is actually the self-intersection set of a smooth immersion in general position then an orientation of one of the curves would induce an orientation on the conjugate pair. The theorem above would tell us how the conjugate pair of a curve must be oriented in relation to the orientation of the original curve.

Another possible application of this necessary condition is to show that the multiple point set of an immersion $f: S^{2} \rightarrow \mathbf{R}^{3}$ in general position cannot be a simple closed 
curve.

Corollary. Let $f: S^{2} \rightarrow \mathbb{R}^{3}$ be a smooth immersion in general position. Then $\Gamma_{f}$, the set of multiple points, cannot be one simple closed curve.

This fact would probably be not too difficult to prove by other methods. Our main purpose here is to demonstrate how the above theorem might be applied in certain situations.

We use a colouring procedure described in Section 1. The proof of the theorem and of the Corollary is given in Section 2.

\section{Colouring the surface}

Throughout the whole section $f: S^{2} \rightarrow \mathbb{R}^{3}$ will be an immersion in general position and $N: S^{2} \rightarrow \mathbb{R}^{3}$ denotes the co-orienting normal, defined as follows. For $p \in S^{2}$ let $u, v \in T_{p} S^{2}$ be tangent vectors such that $\{u, v\}$ is positively oriented. Define $N(p)$ to be the normal vector to the surface $f\left(S^{2}\right)$ at $f(p)$ such that $\left\{f^{\prime}(u), f^{\prime}(v), N(p)\right\}$ is positively oriented in $\mathbb{R}^{3}$.

First we construct a "two-colouring" of $S^{2}-\Gamma_{f}$, that is a function $g: S^{2}-\Gamma_{f}$ $\rightarrow\{0,1\}$ which is constant on the connected components of $S^{2}-\Gamma_{f}$ and assumes different values on neighbouring components.

We start by "two-colouring" the complement of the immersed surface according to whether the winding number of the surface with respect to the given point is even or odd. To be more precise let $x \in \mathbb{R}^{3}-f\left(S^{2}\right)$ and let $\varepsilon>0$ be small enough such that $S_{x}(\varepsilon)$ the sphere of radius $\varepsilon$ around $x$ does not intersect $f\left(S^{2}\right)$. Denote by $\pi_{x, \varepsilon}: \mathbb{R}^{3}-\{x\} \rightarrow S_{x}(\varepsilon)$ the radial projection. Then $\pi_{x, \varepsilon} \circ f: S^{2} \rightarrow S_{x}(\varepsilon)$ is a smooth map and we define the winding number of $f$ at the point $x$ as the degree of this map, that is $w_{f}(x)=\operatorname{deg}\left(\pi_{x, \varepsilon} \circ f\right)$. This is clearly independent of the choice of $\varepsilon$. The "two-colouring" of $\mathbb{R}^{3}-f\left(S^{2}\right)$ is a map $W: \mathbb{R}^{3}-f\left(S^{2}\right) \rightarrow\{0,1\}$ given by

$$
W(x)=w_{f}(x) \bmod 2 .
$$

We call two components $V_{i}$ and $V_{j}$ of $\mathbb{R}^{3}-f\left(S^{2}\right)$ neighbours if there is an open set $D \subset S^{2}$ such that $f(D) \subset \partial V_{i} \cap \partial V_{j}$, where $\partial V_{i}$ denotes the boundary of $V_{i}$. Then the following properties of the winding number are easy to show.

PRopositION 1.1. The functions $w_{f}$ and $W$ are constant on the components of $\mathbb{R}^{3}-f\left(S^{2}\right)$.

If one moves from one connected component to a neighbouring one, then the value of $w_{f}$ and therefore the value of $W$ changes. That is we have:

Proposition 1.2. If $V_{i}$ and $V_{j}$ are neighbouring components of $\mathbb{R}^{3}-f\left(S^{2}\right)$ in the above sense, then $\left.w_{f}\right|_{V_{i}} \neq\left. w_{f}\right|_{v_{j}}$ and the same is true for $W$. 
We now define a "colouring" of the surface. If $p \in S^{2}-\Gamma_{f}$, then $f(p)$ lies on the boundary of exactly two connected components of $\mathbb{R}^{3}-f\left(S^{2}\right)$. Then the co-orienting normal vector $N(p)$ at $f(p)$ points "into" one of the components and we assign the "colour" of that component to $p$.

To be more precise set $U=S^{2}-\Gamma_{f}$. Then $U$ is an open set with the property that each point in $f(U)$ is covered by $f$ exactly once. The "colouring" of $U$ is a map $g: U \rightarrow\{0,1\}$ given as follows. Let $p \in U$ and choose $\varepsilon>0$ small enough such that the curve $\nu(t)=f(p)+t N(p)$ for $t \in(0, \varepsilon)$ does not intersect $f\left(S^{2}\right)$. Then $\nu(t)$ belongs to the same connected component of $\mathbb{R}^{3}-f\left(S^{2}\right)$ for $t \in(0, \varepsilon)$ and define $g(p)=W(\nu(t))$. Since by Proposition $2.1 W$ is constant on the connected components of $\mathbb{R}^{3}-f\left(S^{2}\right)$ the definition does not depend on the value $t \in(0, \varepsilon)$.

The following properties of $g: U \rightarrow\{0,1\}$ follow from the corresponding properties of $W: \mathbb{R}^{3}-f\left(S^{2}\right) \rightarrow\{0,1\}$.

Since the self-intersections of $f\left(S^{2}\right)$ are transversal one can see that $U$ has finitely many open connected components. So we can write $U=\bigcup_{i=1}^{m} U_{i}$, where $U_{i}$ are the connected components.

Proposition 1.3. The map $g: U \rightarrow\{0,1\}$ is constant on the connected components of $U$.

Proof: Let $U_{i}$ be a connected component of $U$ and $p, q \in U_{i}$. Then there is curve $\eta:[0,1] \rightarrow U_{i}$ such that $\eta(0)=p$ and $\eta(1)=q$. Then for a small enough $\varepsilon>0$ the curve $\nu(t)=f(\eta(t))+\varepsilon N(f(\eta(t)))$ does not intersect $f\left(S^{2}\right)$ and $g(p)=W(\nu(0))$ and $g(q)=W(\nu(1))$. This implies that $\nu(0)$ and $\nu(1)$ must belong to the same connected component of $\mathbb{R}^{3}-f\left(S^{2}\right)$, therefore by Proposition 1.1 we have $W(\nu(0))=W(\nu(1))$ and the proof is complete.

We call two connected components $U_{1}$ and $U_{2}$ of $U$ neighbours if $\partial U_{1} \cap \partial U_{2}$ contains an arc. Then we shall show the analog of Proposition 1.2 that $g$ assumes different values on neighbouring connected components.

Proposition 1.4. If $U_{1}$ and $U_{2}$ are neighbouring components of $U \subset S^{2}$ in the above sense, then $\left.g\right|_{U_{1}} \neq\left. g\right|_{U_{2}}$.

PROOF: Since all self-intersections are transversal the normal vectors to the intersecting surfaces are independent. This implies that at any point of the self-intersecting set at most three surfaces can pass through and this can occur only at finitely many points. In other words the number of triple points is finite.

Since $\partial U_{1} \cap \partial U_{2}$ contains an arc it will contain a point $p \in \partial U_{1} \cap \partial U_{2}$ such that $f(p)$ is a double point. Let $p^{\prime} \in S^{2}$ be the conjugate of $p$, that is $f^{-1}(f(p))=\left\{p, p^{\prime}\right\}$.

Let $B_{p}(\varepsilon)$ and $B_{p^{\prime}}(\varepsilon)$ be disjoint small geodesic balls of radius $\varepsilon>0$ in $S^{2}$ centred around the points $p$ and $p^{\prime}$ respectively such that $f: B_{p}(\varepsilon) \rightarrow \mathbb{R}^{3}$ and $f: B_{p}(\varepsilon) \rightarrow \mathbb{R}^{3}$ 
are embeddings.

Then the inverse image of the intersection $f\left(B_{p}(\varepsilon)\right) \cap f\left(B_{p^{\prime}}(\varepsilon)\right)$ by the map $f: B_{p}(\varepsilon) \rightarrow \mathbb{R}^{3}$ is a smooth embedded curve in : $B_{p}(\varepsilon)$ separating $B_{p}(\varepsilon)$ into two parts $B_{p}(\varepsilon) \cap U_{i}$ and $B_{p}(\varepsilon) \cap U_{j}$. Denote this curve by $\eta$. Since $\eta$ is smooth we can select points $q_{1} \in B_{p}(\varepsilon) \cap U_{1}$ and $q_{2} \in B_{p}(\varepsilon) \cap U_{2}$ such that the geodesic $\nu:[0,1] \rightarrow S^{2}$ connecting $q_{1}$ to $q_{2}$ intersects $\eta$ transversally exactly once. Then the curve $f(\nu)$ intersects the surface $f\left(B_{p^{\prime}}(\varepsilon)\right)$ transversally in exactly one point. This remains so if we replace $f(\nu)$ by a curve that is sufficiently close (in the $C^{1}$ topology) to $f(\nu)$. Let $\nu_{\delta}:[0,1] \rightarrow \mathbb{R}^{3}$ be given as $\nu_{\delta}(t)=f(\nu(t))+\delta N(f(\nu(t)))$. Then for a sufficiently small $\delta>0$ we have $g\left(q_{1}\right)=W\left(\nu_{\delta}(0)\right)$ and $g\left(q_{2}\right)=W\left(\nu_{\delta}(1)\right)$. Moreover the curve $\nu_{\delta}[0,1]$ intersects $f\left(B_{p^{\prime}}(\varepsilon)\right)$ transversally in exactly one point and has no other intersections with the rest of $f\left(S^{2}\right)$. This implies that $\nu_{\delta}(0)$ and $\nu_{\delta}(1)$ are in neighbouring connected components of $\mathbb{R}^{3}-f\left(S^{2}\right)$ therefore by Proposition $1.2 W\left(\nu_{\delta}(0)\right) \neq W\left(\nu_{\delta}(1)\right)$ which yields $g\left(q_{1}\right) \neq g\left(q_{2}\right)$. Since by Proposition $1.3 g$ is constant on the connected components $U_{1}$ and $U_{2}$ the proof is complete.

\section{PROOF OF THE THEOREM}

Let us first continue the curve $s$ a little ways beyond the interval $[0,1]$ such that the extended part will not intersect $\Gamma_{f}$. Denoting the extended curve by $s$ as well we now have a curve $s:[0,1+\varepsilon] \rightarrow S^{2}$ such that $s(0)=p_{1}, s(1)=p_{2}$ and the segments $s((0, \varepsilon])$ and $s((1,1+\varepsilon])$ do not intersect $\Gamma_{f}$.

By modifying the curve $s$ in a sufficiently small neighbourhood of the points $p_{1}$ and $p_{2}$ if necessary we may assume without the loss of generality that $s^{\prime}(0)$ and $s^{\prime}(1)$ are orthogonal to $\gamma_{1}^{\prime}\left(t_{0}\right)$ and $\gamma_{2}^{\prime}\left(t_{0}\right)$.

Let us choose an orientation on $S^{2}$ and assume first that $\left\{\gamma_{1}^{\prime}\left(t_{0}\right), s^{\prime}(0)\right\}$ and $\left\{\gamma_{1}^{\prime}\left(t_{0}\right), s^{\prime}(1)\right\}$ are both positively oriented. Let $N_{i}$ for $i=1,2$ be the co-orienting normals at $\gamma_{i}\left(t_{0}\right)$. Let $\alpha$ be the rotation of $\mathbb{R}^{3}$ that leaves the vector $f^{\prime}\left(\gamma_{1}^{\prime}\left(t_{0}\right)\right)$ $=f^{\prime}\left(\gamma_{2}^{\prime}\left(t_{0}\right)\right)$ fixed and rotates $N_{1}$ into $N_{2}$ by an angle less than $\pi$. Then $\alpha$ will carry $f^{\prime}\left(s^{\prime}(0)\right)$ into $f^{\prime}\left(s^{\prime}(1)\right)$. Therefore $N_{s(\varepsilon)}$ and $N_{s(1+\varepsilon)}$, for a sufficiently small $\varepsilon>0$, point into neighbouring connected components of $\mathbb{R}^{3}-f\left(S^{2}\right)$. From the definition of the colouring $g: U \rightarrow\{0,1\}$ we conclude that $s(\varepsilon)$ and $s(1+\varepsilon)$ have different colours; that is $g(s(\varepsilon)) \neq g(s(1+\varepsilon))$. This implies that $s(\varepsilon)$ and $s(1-\varepsilon)$ must have the same colour. Since the colour on the curve $s$ changes every time one passes an intersection point (Proposition 1.4) there have to be an even number of intersection points between $s(\varepsilon)$ and $s(1-\varepsilon)$. Since $\varepsilon$ was chosen small enough such that $s(0, \varepsilon]$ and $s[1-\varepsilon, 1)$ does not intersect $\Gamma_{f}$ we have the same number of intersections between $s(0)$ and $s(1)$ which proves the statement in this case.

The other case when $\left\{\gamma_{1}^{\prime}\left(t_{0}\right), s^{\prime}(0)\right\}$ and $\left\{\gamma_{1}^{\prime}\left(t_{0}\right), s^{\prime}(1)\right\}$ are oppositely oriented 
is very similar. In this case $\alpha$ will carry $f^{\prime}\left(s^{\prime}(0)\right)$ into $-f^{\prime}\left(s^{\prime}(1)\right)$. Therefore either $N_{s(\varepsilon)}$ and $N_{s(1+\varepsilon)}$ or $-N_{s(\varepsilon)}$ and $-N_{s(1+\varepsilon)}$ point into the same connected component of $\mathbb{R}^{3}-f\left(S^{2}\right)$. As a result $s(\varepsilon)$ and $s(1+\varepsilon)$ have the same colour and this implies that $s(\varepsilon)$ and $s(1-\varepsilon)$ have different colours. Applying the same argument as before we conclude that the number of intersections between $s(\varepsilon)$ and $s(1-\varepsilon)$ and therefore between $s(0)$ and $s(1)$ must be odd. The proof of the theorem is complete.

Proof of THE Corollary: First we show that there are two different parameterisations of $\Gamma_{f}$ that are conjugates.

Let $p \in \Gamma_{f}$ be an arbitrary point and let $q \in \Gamma_{f}$ be a conjugate point to $p$. Since the self-intersection of $f$ is transversal at $f(p)=f(q)$ in a small neighbourbood of $p$ and $q$ there are conjugate arcs passing through $p$ and $q$. To be more precise there are curves $\varepsilon_{1,2}:(-\delta, \delta) \rightarrow S^{2}$ with $\varepsilon_{1}(0)=p$ and $\varepsilon_{2}(0)=q$ such that $f\left(\varepsilon_{1}(t)\right)=f\left(\varepsilon_{2}(t)\right)$ for all $t \in(-\delta, \delta)$. Using a continuation argument one obtains two closed curves $\gamma_{1,2}:[0,1] \rightarrow S^{2}$ such that $\gamma_{i}(0)=\gamma_{i}(1)$ for $i=1,2, \gamma_{1}(0)=p, \gamma_{2}(0)=q$ and $\gamma_{1}$ and $\gamma_{2}$ are conjugate curves. Clearly $\Gamma_{f}=\gamma_{1}=\gamma_{2}$ as point sets.

Since $\Gamma_{f}$ is a simple closed curve on $S^{2}$ one can find a curve $s:[0,1] \rightarrow S^{2}$ connecting $p$ and $q$ such that it does not intersect $\Gamma_{f}$ apart from the points $p$ and $q$. Therefore $k_{s}=0$. It is a simple matter to check that $\left\{\gamma_{1}^{\prime}(0), s^{\prime}(0)\right\}$ and $\left\{\gamma_{1}^{\prime}(0), s^{\prime}(1)\right\}$ have different orientation. Therefore the Theorem implies that $k_{s}$ is odd which is a contradiction.

\section{REFERENCES}

[1] H. de Fraysseix and $O$. de Mendez, 'On a characterization of Gauss codes', Discrete Comput. Geom. 22 (1999), 287-295.

[2] C.F. Gauss, Werke VIII (Taubner, Leipzig, 1900).

[3] L. Lovász and M.L. Marx, 'A forbidden subgraph characterization of Gauss codes', Bull. Amer. Math. Soc. 82 (1976), 121-122.

[4] G. Lippner, 'On double points of immersions of spheres', Manuscripta Math. 113 (2004), 239-250.

[5] P. Rosenstiehl, 'Solution algébrique du probléme de Gauss sur la permutation des points d'intersection d'une ou plusieurs coubes fermées du plan', C.R. Acad. Sci. Paris, Sér. A 283 (1976), 551-553.

[6] P. Rosenstiehl, 'A new proof of the Gauss interlace conjecture', Adv. in Appl. Math. 23 (1999), 3-13.

Department of Mathematics and Computer Science

Kuwait University

Safat 13060, Kuwait

e-mail: borbely@ mcs.sci.kuniv.edu.kw 